\title{
Biomimetic tactile target acquisition, tracking and capture
}

\author{
Ben Mitchinson ${ }^{\mathrm{a}, *}$, Martin J. Pearson ${ }^{\mathrm{b}}$, Anthony G. Pipe ${ }^{\mathrm{b}}$, Tony J. Prescott ${ }^{\mathrm{a}}$ \\ ${ }^{a}$ ATLAS Research Group, The University Of Sheffield, UK \\ ${ }^{b}$ Bristol Robotics Laboratory, Bristol, UK
}

\begin{abstract}
Good performance in unstructured/uncertain environments is an ongoing problem in robotics; in biology, it is an everyday observation. Here, we model a particular biological system - hunting in the Etruscan shrewas a case study in biomimetic robot design. These shrews strike rapidly and accurately after gathering very limited sensory information from their whiskers; we attempt to mimic this performance by using model-based simultaneous discrimination and localisation of a 'prey' robot (i.e. by using strong priors to make sense of limited sensory data), building on our existing low-level models of attention and appetitive behaviour in small mammals. We report performance that is comparable, given the spatial and temporal scale differences, to shrew performance, and discuss what this study reveals about biomimetic robot design in general.
\end{abstract}

Keywords: biomimetic, tactile, whiskers, autonomous mobile robotics

\section{Introduction}

An ongoing challenge for robotics is the development of systems that perform well in 'unstructured environments' outside of the laboratory or the warehouse. The key feature of such environments is uncertainty, which has proved difficult to handle using 'conventional' artificial autonomous systems [1], but can be managed effectively using probabilistic techniques [2]. Probabilistic systems, however, tend to be expensive computationally and, what is more, whilst they do provide a formal methodology for representing an uncertain environment, they do not offer recommendations for behaviour. On the other hand, the simplest of biological organisms manage not only to survive but to thrive in such environments, offering an existence proof for simple but effective mechanisms and control. This single observation provides a strong motivation to the roboticist to understand biological systems and to exploit the solutions to which they have converged $[3,4]$ (including to understand how biological solutions relate to probabilistic solutions [5]). This motivation is inspiring entirely novel (or, 'unconventional') approaches to mechanics [6], locomotion [7], sensing [8] and decision-making [9], to give only a handful of recent examples. At the same time, 'embodied' (i.e. robotic) models of biological systems are considered to be particularly useful models in elucidating biological function, owing to their accessibility and completeness [10]. The synergy between these two aspects is clear.

The capabilities of biological systems exceed those of their robotic counterparts in many ways. Amongst these, they exhibit extremely rapid sequences of dynamics, they are able to adapt their behaviour to new

\footnotetext{
* Corresponding author

Email addresses: b.mitchinson@shef.ac.uk (Ben Mitchinson), martin.pearson@brl.ac.uk (Martin J. Pearson), tony.pipe@brl.ac.uk (Anthony G. Pipe), t.j.prescott@shef.ac.uk (Tony J. Prescott)
} 

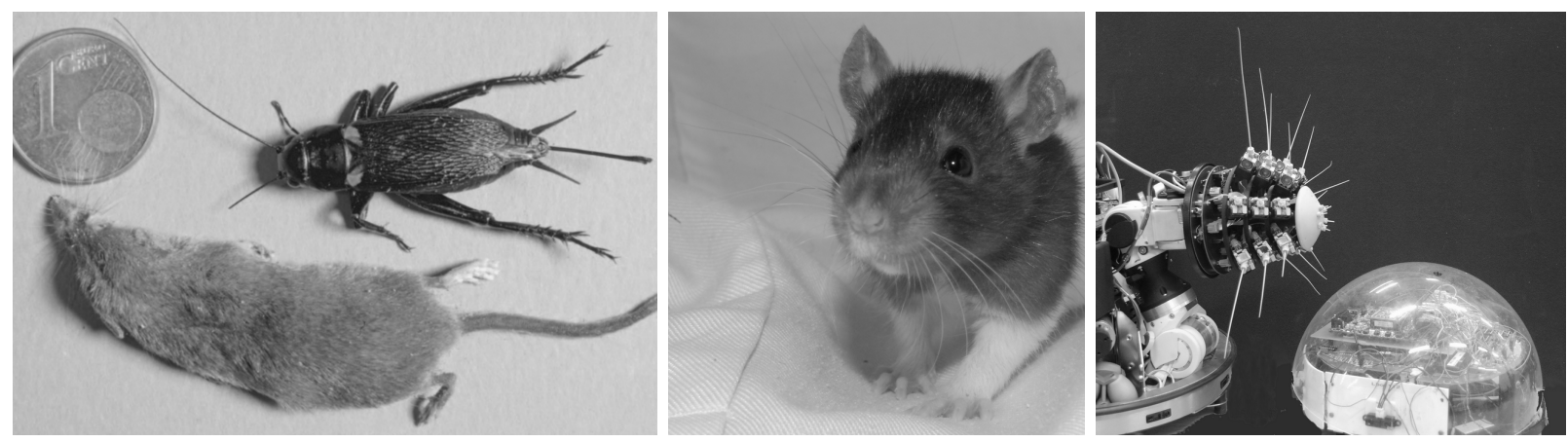

Figure 1: (Left) Shrew and its prey, the cricket (reproduced with permission [12], Copyright (2006) National Academy of Sciences, USA). (Middle) A young rat, showing its large mobile facial whiskers. (Right) The be-whiskered Shrewbot and its hemispherical prey, Preybot.

information on short timescales, and they make highly efficient use of sensing systems that can be quite different from those conventionally employed on robots. Here, we consider a particular biological system that showcases these capabilities in particular, that of prey capture in the Etruscan shrew. This animal must capture prey - sometimes not much smaller than the shrew itself (Figure 1, left) - in twilight or dark conditions [11]. To that end, it relies on large facial 'whiskers' (macrovibrissae), common to many mammals (Figure 1, middle), for both identification and localisation [12]. Whisker-triggered attacks on prey are highly spatially selective and may target an area that has not yet fallen within the whisker sensory field, indicating that they use a 'gestalt-like' representation of the prey, functionally perceiving the whole prey despite sensing just part of it [12]. Overall, this behaviour looks enviable to any roboticist, and we are therefore motivated to understand what is involved in its reproduction.

Many mammals have facial whiskers which are employed for tactile sensing - for a smaller group of whisker specialists, for example including mice, rats, and shrews, which make their living primarily in lowlight or dark conditions, the whiskers are considered to mediate the primary sense of the animal [13]. The whisker sense may be particularly useful in robotics for the same reasons - and in the same conditions - that animals find it useful, though a complete understanding of its capabilities and limitations is still developing. From an engineering perspective, whiskers represent short-range tactile sensors - animals actively move their whiskers, both sweeping them back and forth through the air and repositioning them by moving the head, thus covering the wider sensing space. Previous implementations of artificial whisker sensing, reviewed in $[14,15]$, have shown them to be useful for navigation $[16,17]$ as well as for object identification/localisation $[5,18-20]$. At the same time, robots with whiskers are helping biologists to understand the nature of the task facing biological whisker specialists $[15,21]$.

Below, we report on a robotic model of this prey capture behaviour using a mobile whiskered robot, Shrewbot [15] (Figure 1, right), extending a previous report [22]. Hunting shrews can reach speeds of at least $400 \mathrm{~mm} / \mathrm{s}$ and accelerations of at least $10000 \mathrm{~mm} / \mathrm{s}^{2}$ [23]. The mechanics of Shrewbot support dynamics that are approximately as fast, but Shrewbot $(500 \mathrm{~mm})$ is around 10 times larger than an Etruscan shrew $(50 \mathrm{~mm})$, so that behaviours take around an order of magnitude longer to exhibit. Whilst shrews appear able to rapidly perform reasonably nuanced discriminations [23], Shrewbot was tasked only with discriminating between another mobile robot, Preybot (also pictured, Figure 1, right), and the flat or slightly curved vertical walls of the arena. Nevertheless, our model follows the essentials of the procedure that appears to 
be used by the shrew, expressing the same phases of behaviour, and using a gestalt-like (i.e. model-based) perceptual model to provide most probable identity and location in parallel for an immediate response. We performed two experiments: Experiment 1 measured discrimination performance on the bench, whilst Experiment 2 quantified performance in discrimination/localisation of a stationary or moving target in the open arena. Discrimination performance on the bench for this binary task was almost perfect (perfect where contact was sufficiently robust); this performance was degraded in the open field, but remained fairly reliable. Localisation performance in the stationary case was good, with Preybot reliably located to well within a tenth of its diameter; this performance degraded only a little when the target was moving. Overall, our results illustrate that the shape and surface orientation information carried across the time series recovered from multiple whiskers were sufficient to identify and locate a target in good time to drive accurate and appropriate behavioural responses in this sightless robot. Moreover, they constitute a quantitative analysis of a problem analogous to that faced by the hunting shrew. In our discussion, we identify the potential and shortcomings of this system as a model of biology, and go on to review the system as a case study in biomimetic robotic design.

\section{Methods}

\subsection{Equipment}

Shrewbot's (Figure 1, right) electromechanical architecture is described at length elsewhere [15, 24, 25]. Briefly, it is based on a holonomic mobile platform (Robotinoß, festo.com), has a bespoke three d.o.f. neck (elumotion.com), on the end of which is mounted the head, on which are mounted 18 mobile whiskers (tapered plastic shafts mounted in silicone rubber). Each whisker has 1 d.o.f. (rotation around its base, leading to 'protraction' or forward movement of its tip), and is instrumented for measuring deflection in two axes (denoted $x$ and $y$ ) using a hall-effect sensor and a small powerful magnet glued to the base of the whisker shaft. We can assume, here, that all software components are running at the same rate of $f=20 \mathrm{~Hz}$, leading to response times measured in increments of $T=50 \mathrm{~ms}$ (the much higher frequency data available from the platform are not relevant to this study since we are not discriminating fine spatial or temporal detail such as texture or movement [21]). Sample number is denoted $n \in\{1,2, \ldots\}$.

All motors are configured as position servos (encoders do provide position feedback on this platform, but they are not used for control, here), and all other than those driving the whiskers are controlled by a redundancy-management scheme that is described elsewhere $[10,24,26]$. Briefly, the position of the tip of the 'nose' is controlled, and all other nodes (neck joints, body) are moved the minimum necessary to support the movement of the nose. We have previously argued that this may be a useful model of gross motor control in response to the changing focus of spatial attention in small mammals [25]. In these animals, such as rodents and shrews, much sensory equipment-lips, tongue, teeth, nostrils, and the small sensitive whiskers round the mouth known as micro-vibrissae - is located in this region around the mouth. For these reasons, we use the term 'fovea' to denote the controlled point at the tip of the nose. The whisker motors are controlled individually, according to current behavioural and environmental conditions (see below).

It may be helpful to state clearly that the artificial whiskers of Shrewbot mimic the biological whiskers in a particular property - that is, they are instrumented only at the base, the shaft being made of 'dead' plastic/keratin. This is in contrast to the antennae of some insects, which can be instrumented all along their length. From the deflection vector for the $w$ th whisker $\mathbf{v}_{w}=\left(x_{w}, y_{w}\right)$ we derive a 'contact belief' signal 
$b_{w}=H\left(g_{w}\left(\left\|\mathbf{v}_{w}\right\|-\eta_{w}\right)\right)$, where $\left\|\mathbf{v}_{w}\right\|$ is the magnitude of $\mathbf{v}_{w}, \eta_{w}$ is an estimate of the noise ceiling, $g_{w}$ is a gain, and

$$
H(a)= \begin{cases}0, & a<0 \\ 1, & a>1 \\ a, & \text { otherwise. }\end{cases}
$$

The parameters $g_{w}$ and $\eta_{w}$, which vary with whisker length, are specific to the platform and are not reported; they are chosen such that values of $b_{w}$ of 0 and 1 correspond, approximately and respectively, to 'certainty of not contact' and 'certainty of contact'.

An overhead camera was used whilst mobile experiments were underway to locate both robots with relatively low noise and no integral error in the $2 \mathrm{D}$ world-space defined in our experimental arena. The location of Preybot recovered from the camera, which is assumed to represent the ground truth, is denoted $s_{\mathcal{P}}$. Shrewbot derives an estimate for the location of Preybot at run-time (described below) in egocentric coordinates; post hoc, the location data for Shrewbot recovered from the camera are used to transform this estimate into 2D world-space, where it is denoted $\hat{\mathcal{S}_{\mathcal{P}}}$ (computations on board Shrewbot are performed in relative coordinates, but offline analyses are presented below in absolute arena coordinates, for clarity). Velocity estimates are derived by comparing consecutive location estimates from each series; these estimates are denoted, respectively, $v_{\mathcal{P}}$ (available post hoc) and $\hat{v_{\mathcal{P}}}$ (available to Shrewbot at run-time). $\hat{\boldsymbol{s}_{\mathcal{P}}}$ is boxcarfiltered (one second, causal) and combined with $\hat{v_{\mathcal{P}}}$ assuming a constant prey velocity such that both $\hat{\mathcal{P}_{\mathcal{P}}}$ and $\hat{v_{\mathcal{P}}}$ are smoothed but not lagged. $s_{\mathcal{P}}$ and $v_{\mathcal{P}}$ are not smoothed.

\subsection{Modelled system}

Etruscan shrew prey capture behaviour has been described as consisting of four phases [23]: (i) prehunting, sessile, (ii) search, (iii) contact and (iv) attack (characterised by rapid head movement towards the prey). Whisker movements during phase (ii) [23] are similar to those seen in rat, mouse or opossum during un-motivated exploration [27] - that is, the whiskers are periodically swept forward and then backward in a movement known as 'whisking'. At the same time, the shrew moves rapidly around the environment, providing wide sensory coverage despite the short-range nature of the whiskers as sensors. During phase (iii), whisker movement is modified substantially: movement amplitude decreases whilst protraction setpoint may increase [23]. These results, taken together with similar reports in rat [28], support the hypothesis that the characteristics of whisker movement affect sensor efficacy in a task-dependent way, and that this movement is - accordingly - carefully controlled by the animal. When the shrew commences a strike on its prey, a particular location (the thorax) is targeted in the great majority of cases. Based on several lines of reasoning, but in particular the ability of the shrew to accurately target an area of the prey that has not yet been directly sensed, a gestalt-like representation of the prey has been inferred [12]. In any case, the relative sizes of the prey and the whisker field indicate that only part of the prey can be sensed at any one time (Figure 1, left).

\subsection{Control}

Owing to the morphology of Shrewbot and the size of Preybot (300mm diameter), Shrewbot-like the shrew - cannot sense more than a small part of its prey at any one time. Moreover, it cannot sense 


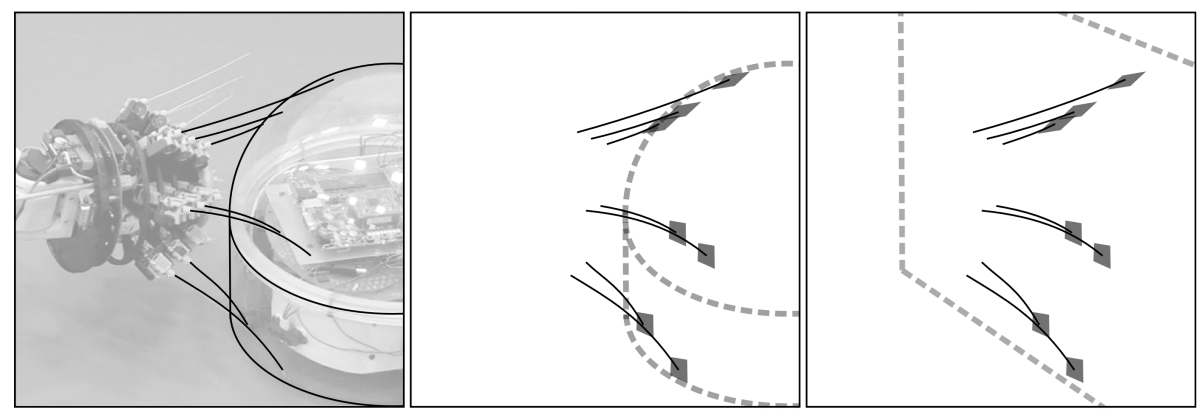

Figure 2: Model-based perception. (Left) Single frame from one trial shows Shrewbot bringing its whiskers forward to make as many contacts with Preybot as possible. Seven contacting whiskers are highlighted, as is Preybot. Models of different percepts (dashed lines) are fitted to the set of 'shapelets' generated from the contact data; Preybot $(\mathcal{P}$, middle) and a vertical wall $(\mathcal{W}$, right). Final model scores are penalised for shapelet location and orientation.

'deeply' enough into Preybot to locate its centre point directly. Fortunately, a model-based approach to prey identification, as suggested by the biology, is able to provide both identity and position estimates simultaneously, despite this paucity of data (see Figure 2). Such an approach requires that a list of possible percepts be pre-defined; here, we assume that the environment offers only one of three possible percepts at each sample time: Preybot $(\mathcal{P})$, a vertical flat or slightly curved wall $(\mathcal{W})$, or nothing $(\mathcal{N})$. This approach can be understood in the terminology of probabilistic perception as the use of 'strong priors'. We assume that a non-zero value of $b_{w}$ for any $w$ indicates contact with something (since sensor noise is accounted for by $\eta_{w}$ ) so that $\mathcal{N} \Longleftrightarrow b_{w}=0 \forall w$. For all other sensory states, only the percepts $\mathcal{P}$ and $\mathcal{W}$ are considered possible. At sample times where such sensory states (some contact) occurred, the following bottom-up/top-down procedure was followed to instantly reach one or other of these percepts.

The bottom-up component of the procedure is the generation of 'shapelets' (oriented fragments of surface [29]) from the instantaneous whisker deflection data. Briefly, this proceeds as follows. First, we assume contact occurs at the whisker tip, essentially for algorithmic simplicity, though tip contact often seems to be the case both in animals [30] and in our experimental conditions. We compute the undeflected whisker tip location, $\mathbf{x}_{\text {undef }}$, geometrically. For each whisker, we measure the gain between whisker tip deflection in two dimensions and the sensory signal $\mathbf{v}_{w}$ on the bench a priori, and use this to estimate tip deflection, $\Delta \mathbf{x}_{\text {def }}$, at run-time, giving the deflected tip location $\mathbf{x}_{\text {def }}=\mathbf{x}_{\text {undef }}+\Delta \mathbf{x}_{\text {def }}$. We compute a direction vector $\Delta \mathbf{x}_{\text {surf }}$ normal to the whisker shaft and to $\Delta \mathbf{x}_{\text {def }}$; this vector is parallel to the set of surfaces that could have led to $\Delta \mathbf{x}_{\text {def }}$ in a first-order frictionless model of tip deflection. In our case, then, the shapelet is actually a set of surfaces defined by $\left\{\mathbf{x}_{\text {def }}, \Delta \mathbf{x}_{\text {surf }}\right\}$ (all those surfaces that pass through the location $\mathbf{x}_{\text {def }}$ and include the surface parallel $\Delta \mathbf{x}_{\text {surf }}$ ). Each shapelet can be considered to be weighted by $b_{w}$ for that whisker, so that non-contacting whiskers, for instance, carry no information in their associated shapelet.

The top-down component of the procedure is the optimisation and appraisal, with respect to the current bottom-up features, of parameterised models of the possible percepts. The model of $\mathcal{P}$ is a $150 \mathrm{~mm}$ radius hemisphere atop a cylindrical base of $100 \mathrm{~mm}$ height; that of $\mathcal{W}$ is a vertical flat surface. Each model has two parameters which together describe the location of the object relative to Shrewbot (the floor of the environment is assumed to be flat and featureless). For $\mathcal{P}$, this is the two-dimensional location on the floor below the centre of the model; for $\mathcal{W}$ it is the distance to and orientation of the vertical wall. The cost for each model, $J$, has two contributions for each contacting whisker $\left(b_{w}>0\right)$ : the first penalises shapelet 
locations $\left(\mathbf{x}_{\text {def }}\right)$ that lay distant from the model surface; the second penalises shapelet surface parallels $\left(\Delta \mathbf{x}_{\text {surf }}\right)$ that do not align with the model surface. At each sample, the initial parametrisations of each model are chosen by simple heuristics $(\mathcal{W}$ is placed at the most forward contact point and oriented to face the robot, $\mathcal{P}$ is placed $150 \mathrm{~mm}$ further forward of the robot), but this particular choice serves only to reduce the computational load of the subsequent optimisation of each model against its unimodal cost function. Following minimisation of $J$ of each model, a time series called 'prey belief' is constructed ${ }^{1}$ from the final cost value for each model (respectively, $J_{\mathcal{P}}(n)$ and $J_{\mathcal{W}}(n)$ ),

$$
b_{\mathcal{P}}(n)=J_{\mathcal{W}}(n) /\left(J_{\mathcal{W}}(n)+J_{\mathcal{P}}(n)\right) \in[0,1] .
$$

The cost function itself has only two free parameters ( $L$, the number of samples over which to aggregate inputs, and $w$, the relative weighting of the second component of the cost function). Our only attempt to tune these parameters was to manually and coarsely adjust $w$ to optimise discrimination performance on the data from Experiment 1 ( $L$ was set, arbitrarily, to 5 , for an aggregation window of $5 T=250 \mathrm{~ms}$ ). See our discussion for more on appraisal of top-down models.

Finally, a unitary percept $U(n)$ (one of $\mathcal{N}, \mathcal{W}$ or $\mathcal{P}$ ) is arrived at for each sample time, and behaviour is driven accordingly. This percept can be written as

$$
U(n)=\left\{\begin{array}{ll}
\mathcal{N}, & b_{w}(n)=0 \forall w \\
\mathcal{P}, & b_{\mathcal{P}}(n) \geq q(n) b_{\mathcal{P}}^{\star} \\
\mathcal{W}, & b_{\mathcal{P}}(n)<q(n) b_{\mathcal{P}}^{\star}
\end{array},\right.
$$

where $b_{\mathcal{P}}^{\star}$ is some discrimination threshold and $q(n)$ is a factor introducing hysteresis to the perceptual decision making, written as

$$
q(n)=\left\{\begin{array}{ll}
0.5, & U(n-1)=\mathcal{P} \\
1, & \text { otherwise }
\end{array} .\right.
$$

In words, where contact of any sort is made, the percept $U(n)$ is that which produces the best match with sensory data, colored by a tendency to stick with the perception of $\mathcal{P}$ once it has been made. This two-way ('bottom-up and top-down') approach to generation of a unitary percept from sensory data is closely related to currently popular hierarchical cortical models (see [31] for a recent review of these in Bayesian terms; see [32] for an application to shape recognition using whiskers).

When $U(n) \neq \mathcal{P}$, Shrewbot exhibits 'explore' behaviour, whereby it makes a series of discrete random moves in a generally forward direction executing a whisk (forward-backward sweep of the whiskers) towards the end of each move. If it contacts something $(U(n)=\mathcal{W}$ ), it investigates it on the next move (an 'orient'), before turning away and exploring in another direction. The details of this simple exploratory behaviour model, the AttenSel model described by Mitchinson et al. [25] which includes an implementation of 'inhibition-of-return' [33], are peripheral to the current study. Here, however, when $U(n)=\mathcal{P}$, a different behaviour is exhibited, which we refer to as 'tracking'. At the next sample time, Shrewbot moves so as to bring Preybot towards the centre of the whisker field on one or other side of the head such that many whiskers can be brought to bear on it (an example of this positioning is shown in Figure 2, left). Since the

\footnotetext{
${ }^{1}$ Note that $b_{\mathcal{P}}(n)$ is undefined (zero over zero) at sample times where $b_{w}=0 \forall w$, but is not needed in these cases.
} 
location of Preybot in the whisker array is controlled (first-order positional control), Shrewbot effectively follows ('tracks') Preybot as it moves during this behaviour. Tracking behaviour continues to be exhibited until either $U(n) \neq \mathcal{P}$, or until a fixed time (arbitrarily set to 4 seconds, for this report) has elapsed since tracking began - this tracking period is intended to allow the kinematic estimates $\hat{s_{\mathcal{P}}}$ and $\hat{v_{\mathcal{P}}}$ to stabilise. In the former case, the encounter is labelled a 'refusal' (R); in the latter case, the encounter is labelled a 'strike' (S). In (S) encounters, a final behaviour is exhibited, denoted 'strike' (see below), and a two-second pause denoted 'hold' follows immediately afterwards. All encounters analysed in Experiment 2 ended as either (R) or (S); a small number of trials were not analysed since no interaction between the robots occurred - these discards are noted, below. An example of this sequence of behaviours is provided below (Figure 5).

In (S) trials, Shrewbot completes the 'prey capture' by 'striking' at the 'strike point' on Preybot, a behaviour that executes in $T_{\mathrm{S}}=0.8 \mathrm{~s}$. The identification of a particular point to be targeted by an 'attack' is in analogy to the behaviour exhibited by the shrew; as for the shrew, the strike point does not need to have been sensed directly in order that it be targeted. The strike point, denoted $\psi$, lies $120 \mathrm{~mm}$ rearward of Preybot's centre, and is marked with a white disc in figures and videos. As it happens, Preybot is radially symmetric, so that $\psi$ can only be identified uniquely if Preybot reveals its orientation by moving; if Preybot is stationary, $\psi$ may lie anywhere around a circle $120 \mathrm{~mm}$ from Preybot's centre. We work around this discrepancy from the biological system by defining an arbitrary point on this circle - that nearest to Shrewbot - as the estimate of $\psi$ when Preybot is stationary. One possible approach to integration across the stationary and moving cases would be to switch between these two behaviours based on the estimated Preybot speed. We chose to avoid a discontinuity in behaviour by targeting a weighted average of these two estimates of the strike point, as follows. If Preybot is estimated to be moving $\left(\left|\hat{v_{\mathcal{P}}}\right| \geq v_{\text {moving }}=\right.$ $80 \mathrm{~mm} / \mathrm{s}$ ), Shrewbot uses its estimates of Preybot's location and velocity at time $t$ to generate an estimate of $\psi\left(t+T_{\mathrm{S}}\right)$, denoted $\hat{\psi}_{1}\left(t+T_{\mathrm{S}}\right)$. If Preybot is estimated to be stationary $\left(\left|\hat{v_{\mathcal{P}}}\right|=0 \mathrm{~mm} / \mathrm{s}\right)$, Shrewbot estimates $\psi\left(t+T_{\mathrm{S}}\right)$ to be $120 \mathrm{~mm}$ along a line from $\hat{s_{\mathcal{P}}}\left(t+T_{\mathrm{S}}\right)$ towards the tip of Shrewbot's snout at time $t$; this estimate is denoted $\hat{\psi}_{0}\left(t+T_{\mathrm{S}}\right)$. When the estimated speed is between these limits, a weighted estimate $\hat{\psi}=\hat{\psi}_{0}+\left|\hat{v}_{\mathcal{P}}\right| / v_{\text {moving }}\left(\hat{\psi}_{1}-\hat{\psi}_{0}\right)$ is used. Shrewbot then executes the 'strike', which places the fovea [25] at $\hat{\psi}\left(t+T_{\mathrm{S}}\right)$ at time $t+T_{\mathrm{S}}$. That is, this behaviour is predictive: the target reached is the expected location of the strike point at the end of the strike period. Thus, a successful strike (rendezvous-ing the fovea on Shrewbot and the strike point on Preybot at the end of the strike period) is the culmination of successful identification, localisation, and velocity estimation. In the stationary case, of course, Shrewbot does not generally target the strike point (white disc) at the end of the strike since $\psi$ cannot be uniquely located.

Whilst expressing explore behaviour, movements of the head and transient protractions of the whiskers more-or-less alternate, so that the robot can be described as exhibiting 'periodic whisking' whilst exploring the world. During tracking behaviour, however, the whiskers undergo constant drive excitation, so that they are held strongly protracted. Working against this, transient negative feedback from $b_{w}$ suppresses the drive to the corresponding whisker, according to the model described by [26]. As previously reported [19], the interaction between this excitation and this suppression often leads to rapid periodic interaction with the surface (something like 'tapping') by contacting whiskers (this can be seen in some of the supplementary videos). This mix of excitatory and inhibitory influences may be a useful model of rat behaviour [34], and may act to maximize the number of contacts that occur whilst normalizing the depth of those contacts. The whisker movement that results during tracking has reduced amplitude and more protracted average angle, 

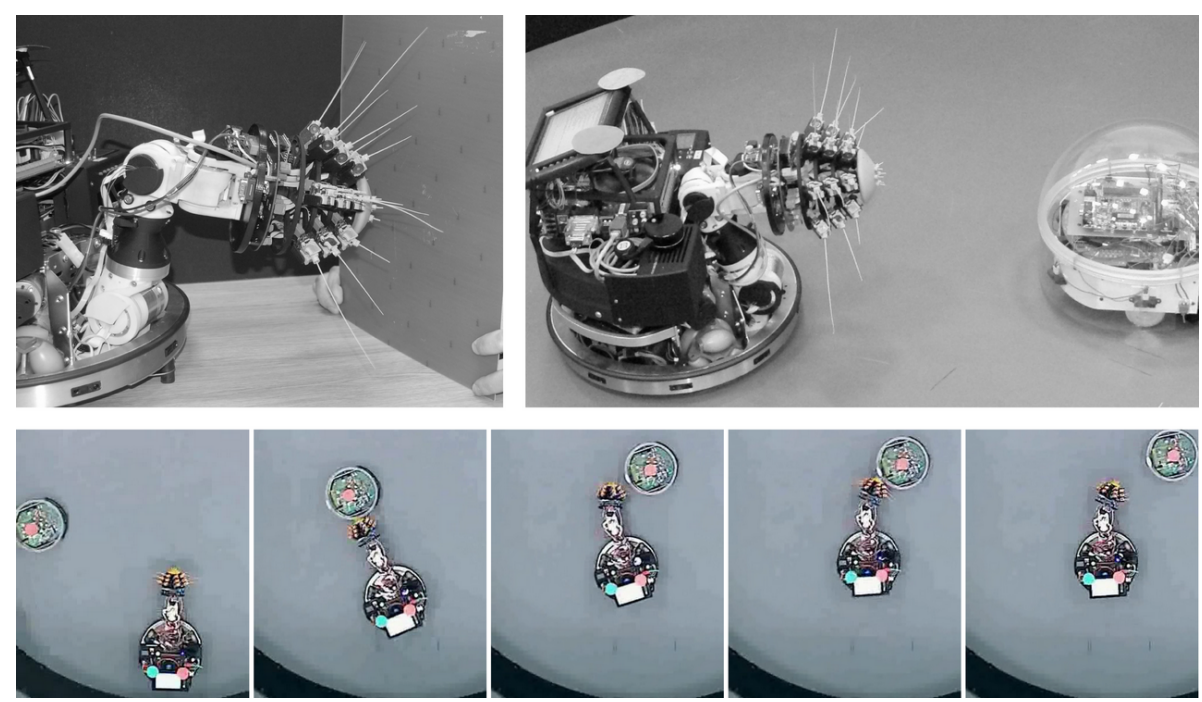

Figure 3: Experimental set-up. (Top left) Experiment 1, experimenter presents stimulus $\mathcal{W}$ to an immobilised Shrewbot. (Top right) Experiment 2, Shrewbot approaches a stationary and helpless Preybot. (Bottom) Series of frames from overhead camera during $(\mathrm{S})$ trial on a moving target. Frame times are $\mathrm{t}=0$ (trial begin), 6.7s (contact), 10.7s (begin strike), 11.5s (complete strike) and 13.5s (trial end).

these being the main features described by Munz et al. [23] and Berg et al. [28].

\section{Results}

It would be possible to reproduce the hunting behaviour of the shrew described above (search, contact, attack) in one experiment. However, the search component of this behaviour would be very time consuming (as well as uninteresting) to reproduce on the robot, as compared with in the animal, owing to the much lower movement rates and lack of auxiliary senses to guide search (such as olfaction and audition). Therefore, we performed two experiments that together assess the remaining characteristics of this behaviour, other than search. In Experiment 1 we assessed discrimination performance on the bench; in Experiment 2 we observed contact, tracking, kinematic estimation and capture behaviour in the arena. In both experiments, the search component of behaviour was rendered unnecessary by preparation of the geometry of each trial (robot-stimulus encounter) by the experimenters. In both experiments, considerable noise was present in the geometry of the first contact between robot and stimulus, owing to the experimental design, as would be expected if the interactions had resulted from random search.

\subsection{Experiment 1: Discrimination}

Experiment 1 was both a test of discrimination performance and our methodology to choose the discrimination threshold $b_{\mathcal{P}}^{\star}$. We placed the robot on the bench and disabled the wheels, but allowed it to move its neck and whiskers freely, as if exploring. The experimenter presented one or other stimulus to the robot by hand $(\mathcal{W}$ or $\mathcal{P}$, Figure 3, top left). These presentations were intended to be varying randomly in location - and, in the case of the vertical wall $\mathcal{W}$, orientation - but were not tightly controlled. On each whisk in the recorded dataset, the stimulus interacted with neither, one, or both whisker fields; the stimulus was stationary during some interactions and moving during others. The control loop was not closed for this 


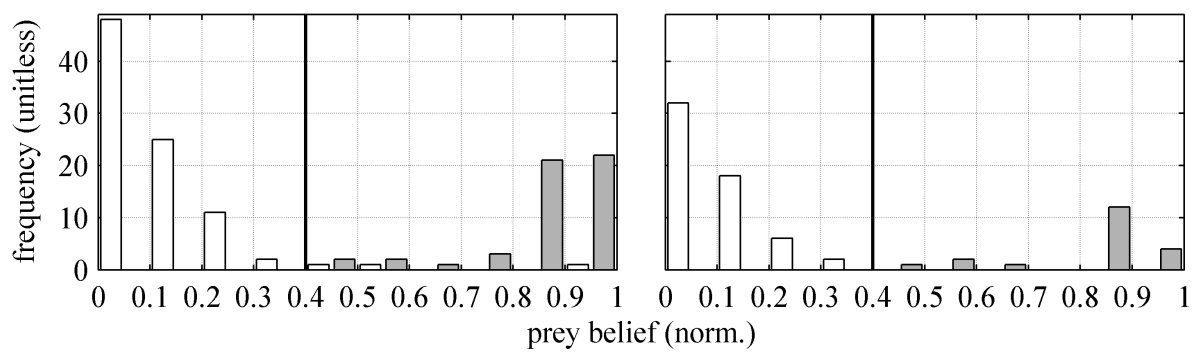

Figure 4: Discrimination summary. Distribution of $b_{\mathcal{P}}$ for (left) all 140 whisks in which contact occurred and (right) only those 78 whisks in which robust or multiple contacts occurred. Data for trials where stimulus was $\mathcal{W} / \mathcal{P}$ are shown using unshaded/shaded bars. Discrimination threshold $b_{\mathcal{P}}^{\star}=0.4$ is shown as solid lines.

experiment - that is, the robot did not change its behaviour in response to the stimuli, and continued to whisk, as if exploring, throughout. 245 whisks were collected, over 11 executions. From these, 140 whisks with some stimulus contact were identified $(89 / 51$ of these were $\mathcal{W} / \mathcal{P})$. A value of $b_{\mathcal{P}}$ was taken from each whisk at the point of maximum whisker protraction. We chose a discrimination threshold of $b_{\mathcal{P}}^{\star}=0.4$, by eye, to classify each stimulus into $\mathcal{W}$ or $\mathcal{P}$; classification performance is summarised in Figure 4 . 3 errors were made over the 140 whisks (all 3 cases were $\mathcal{W}$ being identified as $\mathcal{P}$, i.e. false positive prey identification). Including only those whisks where $\sum_{w} b_{w} \geq 1.0$ (that is, firm contact on a single whisker or some contact on at least two whiskers), 78 whisks were included $(58 / 20$ were $\mathcal{W} / \mathcal{P})$ and no errors were made.

The point of maximum protraction, chosen in this experiment as the point to make a discrimination, occurs shortly after first contact with the stimulus. Thus, the conditions for each trial (whisk) closely mimic the conditions of first contact with a stimulus in the open arena, when the behaving robot would have to respond to the stimulus appropriately. Since the interaction geometry is at its most uncontrolled at this time, before stimulus interaction is exhibited, this condition probably represents a worst case for discrimination performance. As evidence for this proposal, note that in 62 of 140 trials, the condition $\sum_{w} b_{w} \geq 1.0$ was not met, indicating very weak interaction. Following this first interaction, the freely-behaving robot would exhibit one of the 'orient' or 'tracking' behaviours described above, tending to increase the number of whiskers contacting the stimulus, and discrimination performance could therefore reasonably be expected to improve at subsequent sample times.

\subsection{Experiment 2: Localisation}

Shrewbot was positioned roughly in the same position and orientation in each trial, facing 'North' (along the +ve y-axis), and allowed to explore freely. In the first part of the experiment, Preybot was stationary somewhere ahead of Shrewbot (see Figure 3). Three locations were used, each for about one third of trials. In the second part, Preybot was moving on a trajectory that passed ahead of Shrewbot. Two classes of trajectory were used in about half of the trials each, one coming from each side of Shrewbot ${ }^{2}$. Each individual trajectory had (roughly) constant velocity, and a speed of around $100 \mathrm{~mm} / \mathrm{s}$. In those trials in which Shrewbot came into contact with Preybot, behaviour proceeded as described above, resulting either

\footnotetext{
${ }^{2}$ Within each of five conditions (three stationary locations, two non-stationary trajectories) a single random seed was used in all trials, so that an interaction between the robots could be expected with high probability. Notwithstanding this, each individual trial can be considered to be essentially a unique condition: owing to the considerable noise in the initial positioning of the robots in each trial, the exact time and location and configuration of interaction was unpredictable
} 

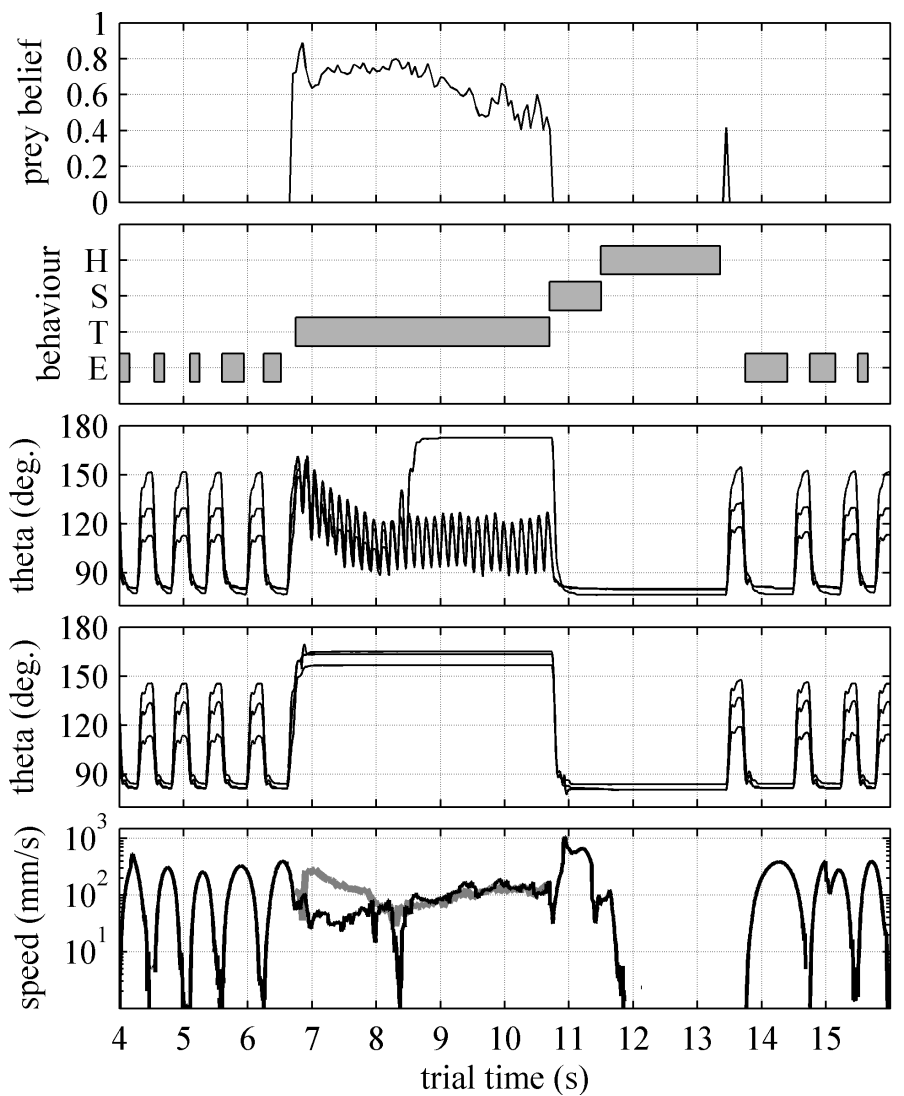

Figure 5: Localisation example ethogram (moving target); each panel is against time within trial. (Panel 1, top) Prey belief, $b_{\mathcal{P}}$. (2) Selected behaviour: (E)xplore, (T)rack, (S)trike, (H)old. (3/4) Whisker angles on the right/left. Preybot was tracked in the right hand side whisker array in this example, and the resulting high frequency oscillations ('tapping' of the contacted surface) in some whiskers are visible in the trace. (5) Estimated prey speed (thick grey, unavailable when prey belief is zero) and Shrewbot snout movement speed (thin black). Towards the end of tracking behaviour, Shrewbot snout is matching estimated speed of prey. 

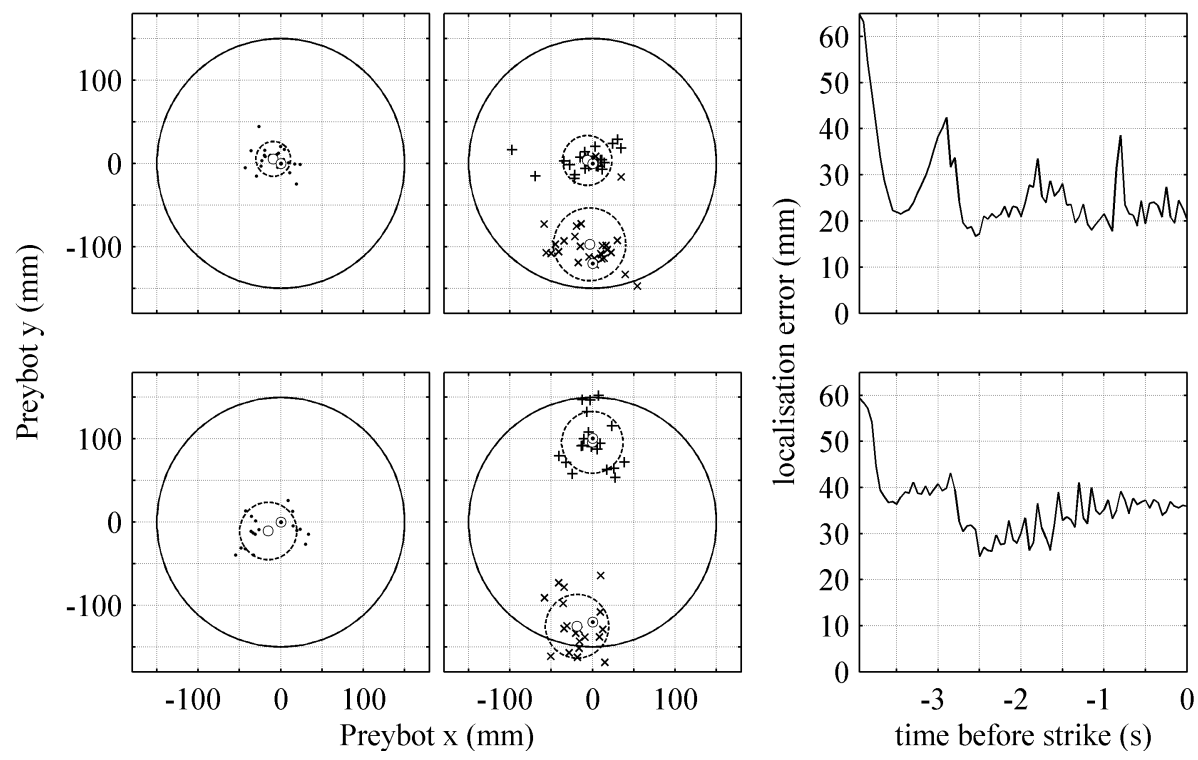

Figure 6: Localisation summary. Two rows of three plots show results for stationary/moving target (upper/lower). The scatterplots present Shrewbot's estimates of Preybot location (•, left-hand column), velocity (+, middle column), and strike point $(x$, middle column), each transformed into a coordinate system centred on Preybot (Preybot extents shown as solid circle). When Preybot is moving, its orientation is known; when it is not, it is assumed to be pointing 'North' (Shrewbot approaches roughly from 'South'). For each estimate, its true value $(\odot)$, measured mean (o) and measured s.d. (dashed circle) is shown. The time series plots (to the right) display the error in Shrewbot's estimate of Preybot location (mean across trials), against time before strike.

in a refusal (R) or a strike (S). Results from an example trial (moving target, $(\mathrm{S})$ trial) are presented in Figure 5; results from all trials in Experiment 2 are summarised in Figure 6. Example overhead videos of trials are available: V1 (goo.gl/6Tq0S, stationary target) and V2 (goo.gl/QCSwR, moving target, same trial as Figure 5 and lower part of Figure 3). Video V3 (goo.gl/HNoIn) is a high-definition example of an (S) trial at $100 \mathrm{~mm} / \mathrm{s} .42$ trials were recorded with Preybot stationary, of which 2 were discarded since the two robots did not interact. Of the remainder, 13 were refusals $(R)$ and 27 were strikes $(S)$, a false negative rate of about 1 in 3 . The standard deviation (root mean square error) across the $\mathrm{S}$ trials of Shrewbot's kinematic estimates were $21 \mathrm{~mm}(23 \mathrm{~mm})$ for position, $30 \mathrm{~mm} / \mathrm{s}(30 \mathrm{~mm} / \mathrm{s})$ for velocity, and $44 \mathrm{~mm}(49 \mathrm{~mm})$ for strike point. 28 trials were recorded with Preybot moving; 2 were discarded since no interaction had occurred. The remainder comprised 7 refusals (R) and 19 strikes (S), a false negative rate of about 1 in 4. Standard deviation (root mean square error) of kinematic estimates were $35 \mathrm{~mm}(38 \mathrm{~mm})$ for position, $37 \mathrm{~mm} / \mathrm{s}(37 \mathrm{~mm} / \mathrm{s})$ for velocity, and $38 \mathrm{~mm}(42 \mathrm{~mm})$ for strike point.

\subsection{Informal Reports}

We informally confirmed the expected correct identification of distractors during mobile search by allowing Shrewbot to approach and contact the arena walls in several unrecorded trials. In most cases, the percept formed following first contact was of $\mathcal{W}$ (correct); in all other cases, the correct percept was formed one or a small number of sample periods afterwards. Following the adoption of the correct percept, Shrewbot moves away from the detected wall to search elsewhere. This informal result is consistent with our understanding of how the reported experiments reflect the wider, but untested, search/hunting behaviour. 
Following the completion of Experiment 2, we attempted to continue the trials using a higher prey speed of $150 \mathrm{~mm} / \mathrm{sec}$. However, it became much more difficult to organise that the robots interact at all, and we were only able to record one instance of tracking and strike in the time available. In this one instance, performance appeared to be similar to results presented above for $100 \mathrm{~mm} / \mathrm{sec}$, but owing to the lack of data it remains unclear whether performance would be reliable at this higher speed. Video V4 (goo.gl/ppdCg) is a high-definition example of an $(\mathrm{S})$ trial at $150 \mathrm{~mm} / \mathrm{s}$.

\section{Discussion}

\subsection{Review}

The experiments reported above constitute a demonstration, using a mobile whiskered (sightless) robot platform, of some key competences observed in the hunting behaviour of a whisker specialist, the Etruscan shrew. The robot exhibits directed random search of its environment. On making whisker contact with a distractor, the robot quickly moves on to search elsewhere. On making whisker contact with a mobile prey robot, the whiskered robot usually ( $\sim 70 \%$ of cases) successfully tracks the robot for a period of time, during which it estimates its kinematics, so that finally it can execute an illustrative 'prey capture' behaviour. In successful (S) trials, localisation accuracy was much finer than the size of the prey robot itself. Across all tested conditions (stationary and moving target) and all three measures (position, velocity, future location of strike point) error can be summarised as being $\sim 30 \mathrm{~mm}$ or $\sim 30 \mathrm{~mm} / \mathrm{s}$, the diameter of Preybot being $300 \mathrm{~mm}$. Altogether, these results illustrate how shape and surface orientation information carried across brief time series recovered from multiple whiskers can be used to identify the what and where of a stimulus in good time to drive behavioural responses. The reported work represents a complete 'whiskered mobile robot system'; that is, a complete and independent physical system that can explore, discriminate different stimuli, and select and execute appropriate behaviour towards them.

The temporal features of shrew hunting, as reported by [23], include periodic whisking near $14 \mathrm{~Hz}$ during search, and an average time between contact and attack of $180 \mathrm{~ms}$. Shrewbot whisks at $\sim 1.5 \mathrm{~Hz}$ during search; whilst we allowed 4 s between contact and attack, graphing kinematic estimates against time (Figures 5 and 6 ) suggests that $1.5 \mathrm{~s}$ of data is more than adequate to stabilise them. Thus, Shrewbot operates around ten times less quickly than the shrew. Changes in direction during attack phase are detected by shrews and drive changes in attack trajectory with a latency as short as 30ms [23]. Shrewbot currently retracts its whiskers during rapid movements (to protect them from damage) so that it cannot detect prey robot acceleration during the 'strike' behaviour; thus, this aspect of the response was not reproduced here. At the Shrewbot timescale, around $300 \mathrm{~ms}$ would be available to detect a change in prey velocity and react accordingly; given the whisking rate during tracking of around $7 \mathrm{~Hz}$, this is enough time for around two additional 'samples', so that reacting at this speed is plausible.

In recent work [25] we have argued that much of the behaviour of small mammalian tactile specialists can be understood as foveation, the fovea in this case being the region around the mouth [37]. This hypothesis can be summarised by the statement that foveation is action for these mammals, since the primary actuators (teeth, tongue) are co-located with the primary sense organs (teeth, lips, tongue, nostrils, microvibrissae). Accordingly, the gross behavioural model presented here (based on [25]) shares the architecture of oculomotor foveation control models - see Figure 7. Lower-level realisations of these oculomotor models achieve control through integration between attention, selection and - thus - identification and assessment of salience [38]. 

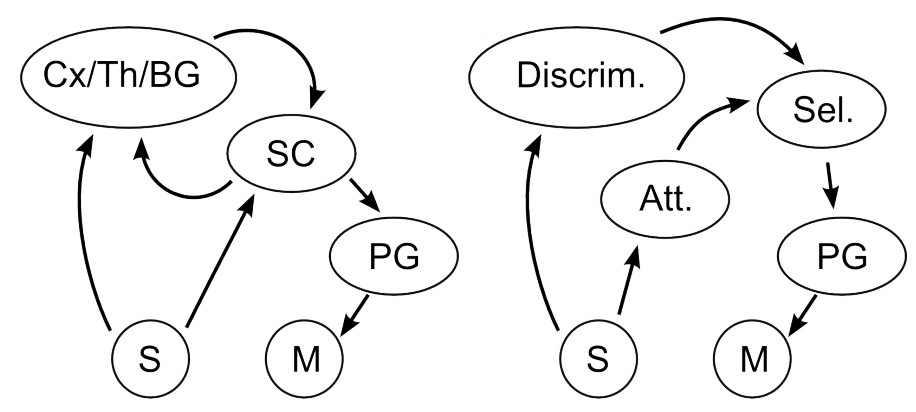

Figure 7: Oculomotor model compared with current model; both panels show Sensory periphery (S), Motor periphery (M), and Pattern Generation (PG). (Left) Essential organisation of contemporary (e.g. [35, 36]) models of oculomotor control. Selection takes place in loops through Superior Colliculus (SC), Cortex, Thalamus and Basal Ganglia (Cx/Th/BG). (Right) Current model shares this architecture but does not represent selection dynamics - rather, hysteresis is used to select between exploratory-like and hunting-like behaviours. Shown are (Discrim)ination, (Att)ention and (Sel)ection.

Available data are consistent with an analogous model of tactile foveation [39, 40]. In our own work, we have used a high-level (functional) model of these integrative processes, with simple hysteretical behaviour selection [25]. The existing model exhibited periodic foveations (as during the explore behaviour, Figure 5). Here, we have extended the model to include a behaviour of continuous foveation to a salient stimulus, which might be described by an observer as 'tracking' behaviour.

We also introduce, here, a simple model of whisker-mediated shape discrimination that is compact but fairly general with respect to stimulus shape and whisker geometry, in the presence of strong priors. The approach utilises a bottom-up/top-down model-based approach to perception (cf. previous approaches to whisker-mediated shape discrimination [41-43]). The only bottom-up features extracted are essentially 'shapelets' (oriented fragments of surface [29]) and therefore the approach promises general discrimination in a mobile condition with any sufficiently-rich whisker geometry. This technique is inspired by the apparent use of a gestalt-like approach by hunting shrews [12] but also likely reflects a more general approach to perception in biological systems [31]. Whilst the model we have developed assumes contact occurs at the tip of the whisker, the extension to contact along the shaft is straightforward provided the contact location can be estimated, and this certainly appears to be possible $[44,45]$. The extension to many percepts in this case is also straightforward both algorithmically and computationally, since each percept is treated as independent.

\subsection{Future directions}

This work represents a first model of hunting behaviour in the Etruscan shrew. Fleshing out the model to more closely mimic the biology will involve several lines of enquiry which may be of interest to biologists and roboticists alike. On the information processing side, one such is to move towards a lower-level description of behavioural selection and implementation, combining electrophysiological data (e.g. [39, 40]) with increasingly rich behavioural data (e.g. [23, 27]) from small mammals, and taking advantage of ongoing interest in models of oculomotor control [46]. This may quickly uncover to what degree snout movements of small tactile mammals are akin, in nature and/or substrate, to saccadic movements in visual mammals, potentially offering both a robust and accessible comparative model to sit alongside the oculomotor system in the biologist's toolbox as well as a component with great potential as the core of a flexible controller for biomimetic autonomous systems. Another is to ask how the biological system might be organised to 
perform the bottom-up/top-down generation of percepts described above. It seems unlikely that parameter optimisation is performed in the biological substrate in an epochal fashion, as described here. And of course, the hand-crafted models used here are unlikely to be optimal; an obvious route to improving performance, as well as increasing the number of stimuli that can be discriminated, is to use experience-driven learning [31]. In the same vein, such an approach could facilitate unsupervised identification of new classes of stimulus, a competence apparently displayed by the shrew when faced with a new class of prey [47]. On the physical side, the development of whiskers that are more robust to damage - without sacrificing sensory performance - would be a substantial leap forward in this area of robotics, and facilitate reproduction of aspects of biological active sensing which remain challenging (such as the very short timescale feedback exhibited by the shrew during a strike [23]). Miniaturisation of this type of hardware also remains a challenge [10]. Finally, the transparency of the tactile sensory representation of shape seems to offer an excellent opportunity for investigation of data fusion across sensory modalities and sub-modalities (such as texture [5]). In particular, the addition of olfaction and/or audition to the robot in the experimental setting described might permit rapid foveation of a target that is outside of the range of the primary sensors, the whiskers, making possible a comprehensive reproduction of the gamut of shrew hunting behaviour.

The experimental paradigm was limited in a number of ways. First, we used a constant Preybot velocity, accordingly assuming a constant velocity in online kinematic estimation. We see no obstacle to the extension to richer kinematics, for instance using a conventional Kalman filter for estimation, with the expected effects on accuracies at different prediction horizons. Experimental analysis would require a far larger number of trials, however, to adequately cover a larger kinematic space, so that data collection would probably be eased by the addition of supplementary sensors as outlined above. Second, Preybot speed was limited to $100 \mathrm{~mm} / \mathrm{sec}$, comparable to Shrewbot speed during exploration. The computations required of this system are relatively lightweight, so the practical constraints on increasing the speed of operation would be physical. In particular, the limit may be down to the whiskers, the self-induced movement signals from which increase as the actuation speeds of the robot and/or whiskers increase. Along with physical robustness, this suggests two directions in which the development of physical whisker sensors might proceed. This aside, we see no reason why the system would not operate well at speeds substantially higher than that used in this report. Third, Preybot was constrained to move in two dimensions. Shrewbot processes information in three dimensions so we would anticipate that the system described would operate correctly without modification were Preybot free to move in this way. Finally, the strategy of measuring motion (including the value of the parameter $v_{\text {moving }}$ ) to orient the prey model and, thus, identify the 'strike point' was chosen as a consequence of Preybot's radial symmetry, and is not a requirement of this approach to control. Shrews will make accurate attacks on stationary crickets and attacks seem to be targeted, rather, through recognition of body parts [12]. A future investigation might select and orient the model of the prey by feature recognition, rather than by motion measurement.

\subsection{Biomimetic design}

What impact has the biomimetic approach had on the design of this robot? First, the design of the primary sense of this robot - which is able to locate, identify, and (notionally) capture a target without the use of any form of radiation - has been driven entirely by observations of small mammals [14], which operate effectively in complete darkness using their facial whiskers [12]. Second, the model of motor control used by the robot is based on our model of body movement in small mammals. That is, that all joint movements 
serve to support movement of a fovea (located near the mouth), providing a unique kinematic solution in the presence of motor plant redundancy that is both effective and computationally straightforward [10, 24, 26]. Third, foveation targets are driven by attention in animals [33]; we have co-opted the essentials of this model, resulting in behaviour that both is bio-plausible to the observer and-more importantly-serves effectively as a basis for more abstract behaviour (Mitchinson et al. [25] and this report). Fourth, borrowing the gestalt-like approach to perception inspired by shrew hunting behaviour, we have found a rapid and reliable shape discriminator applicable for online use in a resource-limited environment (this report).

But - what use a robot that pretends to hunt shrews? First, the processing of whisker signals has proved computationally inexpensive; this corresponds to the fact that the measurements (physical interactions) and the solutions (shapes, textures) exist in essentially the same space (physical space), avoiding the myriad ambiguities and signal transforms that are so prevalent in visual processing systems. In this sense, whisker touch is showing itself to be superior to vision as a robotic sensory system. By this we do not, of course, mean to undermine the common understanding that robots - just like animals - benefit from multi-modal sensing. On the contrary: we noted above in the description of Experiment 2 that Shrewbot would have taken unreasonably long to make first contact with Preybot without help from the experimenters; not so the shrew, aided by olfaction and audition even when hunting in the dark. Second, a highly visible feature of biological designs is their flexibility. To take a relevant example, management of attention does not just enable hunting, but also fleeing, fighting, scavenging, and so on. Indeed, in an agent for which foveation is action [25] — and useful robots of this class can easily be imagined - management of attention is tantamount to action selection. Thus, we consider that an attention management system has the potential to be a critical core component of future autonomous systems. Similarly, a gestalt-like perception competence provides the substrate not just for the reliable identification of crickets, but for the development of a comprehensive and practically-useful description of the world at levels of abstraction as removed from the periphery as necessary $[31]$.

Coming back to the challenge we started with: why might a system designed in this way prove useful in an unstructured or uncertain environment? The answer to this question might be in the observations we made back in Section 1 of the positive competences of biological systems: that they exhibit extremely rapid sequences of dynamics, are able to adapt their behaviour to new information on short timescales, and make highly efficient use of their sensing systems. If by co-opting the underlying characteristics of biological 'designs' we can achieve similar efficiency, flexibility and scalability, then there is the potential to achieve similar performance in these challenging environments.

\subsection{Acknowledgments}

The authors would like to thank Jason Welsby and Harsimran Singh (Bristol Robotics Laboratory) for their contributions to Shrewbot and Preybot, respectively. Supported by the FP7 grant BIOTACT (ICT-215910).

[1] D. Nitzan. Development of intelligent robots: achievements and issues. Robotics and Automation, IEEE Journal of, 1(1): $3-13,1985$.

[2] S Thrun, W Burgard, and D Fox. Probabilistic robotics. The MIT Press, 2006.

[3] J. Krichmar and H. Wagatsuma. Neuromorphic and Brain-Based Robots. Cambridge University Press, 2011.

[4] K. H. Low, S. Guo, X. Deng, R. Vaidyanathan, J. Tangorra, H. C. Park, and F. Iida. Editorial for special issue-special issue on focused areas and future trends of bio-inspired robots:" analysis, control, and design for bio-inspired robotics". Journal of Robotics and Mechatronics, 24(4):559, 2012. 
[5] N. F. Lepora, C. W. Fox, M. H. Evans, M. E. Diamond, K. Gurney, and T. J. Prescott. Optimal decision-making in mammals: insights from a robot study of rodent texture discrimination. Journal of The Royal Society Interface, 9(72): $1517-1528,2012$.

[6] H. G. Marques, M. Jantsch, S. Wittmeier, O. Holland, C. Alessandro, A. Diamond, M. Lungarella, and R. Knight. Ecce1: the first of a series of anthropomimetic musculoskeletal upper torsos. In Humanoid Robots (Humanoids), 2010 10th IEEE-RAS International Conference on, pages 391-396. IEEE, 2010.

[7] P. Liljebäck, KY Pettersen, Ø. Stavdahl, and JT Gravdahl. A review on modelling, implementation, and control of snake robots. Robotics and Autonomous Systems, 60(1):29-40, 2012.

[8] B. B. Edin, L. Ascari, L. Beccai, S. Roccella, J. J. Cabibihan, and MC Carrozza. Bio-inspired sensorization of a biomechatronic robot hand for the grasp-and-lift task. Brain research bulletin, 75(6):785-795, 2008.

[9] T. Schmickl, R. Thenius, C. Moeslinger, G. Radspieler, S. Kernbach, M. Szymanski, and K. Crailsheim. Get in touch: cooperative decision making based on robot-to-robot collisions. Autonomous Agents and Multi-Agent Systems, 18(1): 133-155, 2009.

[10] Ben Mitchinson, Martin J Pearson, Anthony G Pipe, and Tony J Prescott. Biomimetic robots as scientific models: A view from the whisker tip. In Jeffrey L Krichmar and Hiroaki Wagatsuma, editors, Neuromorphic and brain-based robots, pages 23-57. Cambridge University Press, 2011.

[11] C. Roth-Alpermann and M. Brecht. Vibrissal touch in the etruscan shrew. Scholarpedia, 4(11):6830, 2009.

[12] F. Anjum, H. Turni, P. G. Mulder, J. van der Burg, and M. Brecht. Tactile guidance of prey capture in etruscan shrews. Proc Natl Acad Sci USA, 103(44):16544-16549, 2006.

[13] Tony J Prescott, Robyn A Grant, and Ben Mitchinson. Vibrissal behavior and function. Scholarpedia, 6(10):6642, 2011.

[14] T. J. Prescott, M. J. Pearson, B. Mitchinson, J. C. W. Sullivan, and A. G. Pipe. Whisking with robots: from rat vibrissae to biomimetic technology for active touch. IEEE Robotics and Automation Magazine, 16(3):42-50, 2009.

[15] Martin J Pearson, Ben Mitchinson, J Charles Sullivan, Anthony G Pipe, and Tony J Prescott. Biomimetic vibrissal sensing for robots. Phil. Trans. R. Soc. B, 366:3085-3096, 2011.

[16] M. Fend, H. Yokoi, and R. Pfeifer. Optimal morphology of a biologically-inspired whisker array on an obstacle-avoiding robot. Advances in Artificial Life, pages 771-780, 2003.

[17] C. Fox, M. Evans, M. Pearson, and T. Prescott. Tactile slam with a biomimetic whiskered robot. In Robotics and Automation (ICRA), 2012 IEEE International Conference on, pages 4925-4930. IEEE, 2012.

[18] M. J. Pearson, A. G. Pipe, C. Melhuish, B. Mitchinson, and T. J. Prescott. Whiskerbot: A robotic active touch system modeled on the rat whisker sensory system. Adaptive Behaviour, 15(3):223-240, 2007.

[19] J. C. Sullivan, B. Mitchinson, M. J. Pearson, M. Evans, N. F. Lepora, C. W. Fox, C. Melhuish, and T. J. Prescott. Tactile discrimination using active whisker sensors. Sensors Journal, IEEE, 12(2):350-362, 2012.

[20] J. H. Solomon and M. J. Hartmann. Biomechanics: Robotic whiskers used to sense features. Nature, 443(7111):525, 2006.

[21] C. W. Fox, B. Mitchinson, M. J. Pearson, A. G. Pipe, and T. J. Prescott. Contact type dependency of texture classification in a whiskered mobile robot. Autonomous Robots, 26(4):223-239, 2009.

[22] B. Mitchinson, M. Pearson, A. Pipe, and T. Prescott. Predictive prey pursuit in a whiskered robot. Advances in Autonomous Robotics, pages 343-353, 2012.

[23] M. Munz, M. Brecht, and J. Wolfe. Active touch during shrew prey capture. Frontiers in behavioral neuroscience, 4(191), 2010.

[24] Martin J Pearson, Ben Mitchinson, Jason Welsby, Tony Pipe, and Tony J Prescott. Scratchbot: Active tactile sensing in a whiskered mobile robot. In Proceedings of From Animals To Animats 11, Paris, 25-29 August, volume 6226 of LNCS, pages $93-103,2010$.

[25] Ben Mitchinson, Martin J Pearson, Anthony G Pipe, and Tony J Prescott. The emergence of action sequences from spatial attention: insight from mammal-like robots. In Proceedings of Living Machines, 9-12 July, Barcelona (submitted), 2012.

[26] Ben Mitchinson, Martin Pearson, Chris Melhuish, and Tony J. Prescott. A model of sensorimotor co-ordination in the rat whisker system. In From Animals to Animats 9: Proceedings of the Ninth International Conference on Simulation of Adaptive Behaviour, pages 77-88, 2006.

[27] Ben Mitchinson, Robyn A Grant, Kendra Arkley, Vladan Rankov, Igor Perkon, and Tony J Prescott. Active vibrissal sensing in rodents and marsupials. Phil. Trans. R. Soc. B, 366:3037-3048, 2011.

[28] R. W. Berg and D. Kleinfeld. Rhythmic whisking by rat: Retraction as well as protraction of the vibrissae is under active muscular control. J Neurophys, 89(1):104-117, Jan 2003. doi: 10.1152/jn.00600.2002.

[29] CW Fox, MH Evans, MJ Pearson, and TJ Prescott. Towards hierarchical blackboard mapping on a whiskered robot. Robotics and Autonomous Systems, 2012.

[30] T. J. Prescott, B. Mitchinson, P. Redgrave, C. Melhuish, and P. Dean. Three-dimensional reconstruction of whisking patterns in freely moving rats. Society For Neuroscience, November 12-16, Poster 625.3, 2005.

[31] W. Penny. Bayesian models of brain and behaviour. ISRN Biomathematics, 2012, 2012.

[32] A. Motiwala, C. Fox, N. Lepora, and T. Prescott. Sensing with artificial tactile sensors: an investigation of spatio-temporal inference. Towards Autonomous Robotic Systems, pages 253-264, 2011.

[33] Laurent Itti and Christof Koch. Computational modelling of visual attention. Nature Reviews Neuroscience, 2:194-203, 2001.

[34] Ben Mitchinson, Chris J. Martin, Robyn A. Grant, and Tony J. Prescott. Feedback control in active sensing: rat exploratory whisking is modulated by environmental contact. Royal Society Proceedings B, 274(1613):1035-1041, 2007.

[35] K. Arai, E. L. Keller, and J. A. Edelman. Two-dimensional neural network model of the primate saccadic system. Neural networks, 7(6-7):1115-1135, 1994.

[36] J. M. Chambers, K. Gurney, M. Humphries, and A. Prescott. Mechanisms of choice in the primate brain: a quick look at 
positive feedback. In Modelling Natural Action Selection: Proceedings of an International Workshop, pages 45-52, 2005.

[37] M. Brecht, B. Preilowski, and M. M. Merzenich. Functional architecture of the mystacial vibrissae. Behavioural Brain Research, 84:81-97, 1997.

[38] A. Straube and U. Büttner, editors. Neuro-ophthalmology: neuronal control of eye movements. S Karger Ag, 2007.

[39] N Sahibzada, P Dean, and P Redgrave. Movements resembling orientation or avoidance elicited by electrical stimulation of the superior colliculus in rats. J Neurosci, 6(3):723-733, 1986

[40] M. E. Hemelt and A. Keller. Superior sensation: superior colliculus participation in rat vibrissa system. BMC Neuroscience, 8:12, 2007.

[41] R. A. Russell and J. A. Wijaya. Object location and recognition using whisker sensors. In Australian Conference on Robotics and Automation. Citeseer, 2003.

[42] D. Kim and R. Möller. Biomimetic whiskers for shape recognition. Robot Autonom Syst, 55(3):229-243, 2007.

[43] C. Fox, M. Evans, J. Stone, and T. Prescott. Towards temporal inference for shape recognition from whiskers. In Proceedings of Towards Autonomous Robotic Systems (TAROS), 2008.

[44] M. Szwed, K. Bagdasarian, B. Blumenfeld, O. Barak, D. Derdikman, and E. Ahissar. Responses of trigeminal ganglion neurons to the radial distance of contact during active vibrissal touch. $J$ Neurophys, 95:791-802, 2006.

[45] J. A. Birdwell, J. H. Solomon, M. Thajchayapong, M. A. Taylor, M. Cheely, R. B. Towal, J. Conradt, and M. J. Z. Hartmann. Biomechanical models for radial distance determination by the rat vibrissal system. J Neurophys, 98:2439$2455,2007$.

[46] Neeraj J Gandhi and Husam A Katnani. Motor functions of the superior colliculus. Annu. Rev. Neurosci., 34:205-31, 2011.

[47] F. Anjum and M. Brecht. Tactile experience shapes prey-capture behavior in etruscan shrews. Frontiers in Behavioral Neuroscience, 6, 2012 